\title{
Inventário de valores organizacionais
}

\author{
Alvaro Tamayo \\ Ana Magnólia Mendes \\ Maria das Graças Torres da Paz
}

Universidade de Brasília

\begin{abstract}
Resumo
Foram postuladas três dimensões bipolares para representar os valores organizacionais. Um instrumento composto por 36 itens foi administrado a uma amostra de 1010 empregados de uma empresa pública. Os dados foram submetidos a análise multidimensional (ALSCAL), utilizando o modelo euclidiano. As medidas de fit foram: s-stress $=0,18319$, Stress $=0,15985$ e RSQ $=0,88563$. As três dimensões postuladas (autonomia versus conservadorismo, hierarquia versus igualitarismo e domínio versus harmonia) foram confirmadas pela disposição dos valores no espaço semântico. Os coeficientes alpha foram superiores a 0,80 com exceção do alpha para conservadorismo que foi 0,77 .

Palavras-chave: Valores, Valores organizacionais, Psicometria, Diagnóstico organizacional
\end{abstract}

\footnotetext{
Abstract

Organizational values inventory

Three bipolar dimensions were postulated to represent the organizational values. An instrument, composed of 36 items, was administered to a sample of 1010 employees of a public enterprise. The data were submitted to multidimensional
} 
Key words:

Values, Organizational values,

Psychometrics, Organizational analysis analysis (ALSCAL), using the Euclidean model. Measures of fit were: $\mathrm{s}$-stress $=.18319$, Stress $=.15985$, and RSQ $=.88563$.

The value disposition in the semantic space confirmed the three postulated dimensions: autonomy versus conservation, hierarchy versus equalitarianism, and mastery versus harmony. Coefficients alpha were above .80, with the exception of the alpha for conservatism factor that was .77.

Compared to the distant influence of culture, the proximal influences of the organizations where we work would seem at first blush to be more potent. Surprisingly, then, there have been few attempts to measure organizational values in a systematic manner (Bond, 1997, p. 260).

Toda empresa cria sua própria cultura, seu próprio clima de trabalho, com crenças, tradições, usos, rituais, rotinas, normas, valores e tabus próprios. A Psicologia Organizacional atual dá grande importância ao estudo da cultura organizacional por considerar que ela é determinante do desempenho individual, da satisfação no trabalho e da produtividade da empresa. Segundo Rowlinson e Hassard (1993), o interesse continuado na literatura internacional em torno ao conceito de cultura organizacional e ao estudo do impacto da mesma sobre o comportamento dos empregados sugere uma mudança na ênfase no estudo das organizações. Segundo Schein (1990), "A cultura será, para a Psicologia Organizacional, um conceito de importância crescente. Sem ele, não nos seria possível compreender a mudança e a resistência à mudança. Quanto maior o nosso empenho em ajudar as organizações a desenhar as suas estratégias fundamentais, particularmente na área de recursos humanos, tanto mais importante será poder ajudar as organizações a decifrar a suas próprias culturas” (p. 117).

A fonte da cultura em geral são as exigências universais do ser humano. Essas exigências são basicamente de três tipos: as necessi- 
dades biológicas do organismo, as necessidades sociais relativas à regulação das interações interpessoais e as necessidades sócioinstitucionais referentes à sobrevivência e bem-estar dos grupos (Kluckhon, 1951; Rokeach, 1973; Schwartz \& Bilsky, 1987, 1990). O indivíduo, para poder dar conta da realidade, tem que reconhecer essas necessidades e planejar, criar ou aprender respostas apropriadas para a sua satisfação. Essa satisfação, porém, deve acontecer através de formas aceitáveis para o resto do grupo. Desta forma, surgem os valores que são princípios e metas que norteiam o comportamento do indivíduo.

Uma organização não está constituída por partes físicas, mas por estruturas de eventos, de interações, de atividades por ela executadas (Allport, 1962; Schein, 1965). O prédio onde funciona, as pessoas que a integram e o equipamento técnico que utiliza não constituem a sua estrutura. A sua verdadeira estrutura é constituída pelo seu próprio funcionamento. Segundo Katz e Kahn (1978), os principais componentes de uma organização são os papéis, as normas e os valores. Estes três elementos definem e orientam o funcionamento da empresa. Os papéis definem e prescrevem formas de comportamento associadas a determinadas tarefas, as normas são expectativas transformadas em exigências e os valores "são as justificações e aspirações ideológicas mais generalizadas” (Katz \& Kahn, 1978, p. 54). Os papéis diferenciam as funções e os cargos exercidos pelos indivíduos e as normas e os valores são elementos integradores, no sentido de que eles são compartilhados por todos ou por boa parte dos membros de uma organização.

A ênfase na cultura em geral e na cultura organizacional em particular é crescente na literatura internacional. É de capital importância, portanto, elaborar teorias ou modelos da cultura organizacional e estratégias que permitam a elaboração de perfis culturais das organizações. Um passo nesta direção pode ser o estudo dos valores organizacionais. Eles constituem o núcleo fundamental da cultura organizacional (De Freitas, 1994; Deal \& Kennedy, 1988; Hofstede, 1980; Hofstede, Neuijen, Ohayv, \& Sanders, 1990; Peters \& Waterman, 1982; Schwartz \& Ros, 1995; Tamayo, 1998; Triandis, 1994, 1995). A "cultura corporativa é um padrão de crenças e valores compartilha- 
dos", afirma Davis (1984, p. 1). Para Kotter e Heskett (1994), a cultura organizacional é definida pelos valores compartilhados pelas pessoas em um grupo.

Tradicionalmente, duas abordagens têm sido utilizadas para identificar e avaliar os valores organizacionais. A primeira, estuda os valores a partir de documentos oficiais da empresa (relatórios anuais, estatutos, discursos etc.). A maioria dos estudos utilizando esta abordagem é de tipo qualitativo. Uma contribuição significativa que integra o qualitativo e o quantitativo foi dada por Kabanoff, Waldersee e Cohen (1995). Eles procuraram em revistas internas, em pronunciamentos sobre a missão e nos relatórios anuais de 88 empresas australianas, referências a valores (autoridade, comprometimento, liderança, produtividade, eficiência etc.). As diferenças na frequiência relativa com que as empresas faziam referência a esses valores, foram utilizadas para classificar as empresas em grupos axiológicos diferentes. Recentemente, Kabanoff e Daly (2000), utilizando a mesma estratégia e análises estatísticas mais sofisticadas, desenvolveram uma tipologia de valores organizacionais composta por duas dimensões: a estrutura e o processo da organização. Essas duas dimensões dão origem a quatro tipos: Elite, Liderança, Meritocrática e Participativa. O maior inconveniente da abordagem que utiliza documentos da empresa para avaliar os valores organizacionais é que, freqüentemente, existe incongruência entre os valores estabelecidos no papel e os que são realmente praticados na organização. A segunda alternativa tradicional para o estudo dos valores organizacionais consiste em utilizar a média dos valores individuais dos membros da organização como estimativa dos valores organizacionais (Sego, Hui \& Law, 1997). Como freqüentemente existe incongruência entre os valores pessoais dos empregados e os valores organizacionais, o resultado obtido não expressa de forma adequada os valores da organização. Este é o maior inconveniente desta abordagem.

Nesta pesquisa, propõe-se uma abordagem diferente que consiste em estudar os valores organizacionais a partir da percepção que os empregados têm dos valores existentes e praticados na sua empresa. No discurso quotidiano dos empregados freqüentemente podem ser 
observadas análises diretas dos valores da sua organização ou alusões indiretas aos mesmos. Os empregados têm uma visão relativamente clara dos valores que predominam na sua organização. Essa percepção, seja ela correta ou errada, influencia o seu comportamento (Sego et al., 1997). Ao analisar os valores organizacionais a partir da percepção dos empregados é como se o pesquisador estivesse utilizando observadores internos, envolvidos ativamente no processo coletivo, para identificar as prioridades axiológicas da organização.

Todo empregado é capaz de identificar valores que predominam na sua organização e até detectar diferenças nos valores entre setores da empresa ou entre a sua empresa e outras que ele conhece. Uma organização pode ser descrita como sendo norteada por valores referentes aos aspectos técnico e administrativo, ao passo que uma outra pode ser percebida como colocando a ênfase no extremo oposto, centrando suas preocupações principalmente no bem-estar dos indivíduos que a compõem. A percepção dos valores de uma organização parece não ter sido objeto de estudo sistemático. Uma explicação para esta situação pode ser a ausência de instrumentos de medida adequados para avaliar a percepção dos valores organizacionais.

Como descrever ou definir os valores organizacionais? Tamayo (1996) os define como princípios ou crenças, organizados hierarquicamente, relativos a estados de existência ou a modelos de comportamento desejáveis que orientam a vida da empresa e estão ao serviço de interesses individuais, coletivos ou mistos. Tamayo e Gondim (1996) salientam vários aspectos nesta definição: os aspectos cognitivo e motivacional, a função dos valores e a organização hierárquica dos mesmos.

1) O aspecto cognitivo dos valores organizacionais, constitui um elemento básico, já que eles são crenças existentes na empresa, isto é, formas de conhecer a realidade organizacional, respostas cognitivas prontas e privilegiadas a problemas organizacionais. Essas crenças podem ser relacionadas com diversas dimensões da vida organizacional, tais como a produção, a qualidade, as interações interpessoais, o respeito à autoridade gerencial, a obediência às normas etc. Os valores são crenças valorizadas, enfatizadas na vida 
organizacional. Nem toda crença constitui um valor, somente aquelas que são enfatizadas e que são socialmente aceitas como princípios que orientam a vida da organização. Obviamente, as crenças que constituem os valores estão em interação entre si e com outras crenças, de forma a constituir um sistema de valores complexo e organizado hierarquicamente. Os valores funcionam como padrões para o julgamento e a justificação do comportamento de si e dos outros.

2) O aspecto motivacional. A raiz dos valores organizacionais é motivacional, eles expressam interesses e desejos de alguém. Esse alguém pode ser um indivíduo, o dono, o fundador, um gerente ou alguma pessoa influente na empresa; pode também ser um grupo, o conjunto dos membros da empresa. Os valores podem expressar interesses tanto do indivíduo como da coletividade. Assim, os valores organizacionais representam metas mais ou menos conscientes da empresa (Tamayo \& Schwartz, 1993). "They can motivate action, giving it direction and emotional intensity" (Schwartz, 1994, p. 21).

3) A função dos valores. Os valores têm como função orientar a vida da empresa, guiar o comportamento dos seus membros. Eles são determinantes da rotina diária na organização, já que orientam a vida da pessoa e determinam a sua forma de pensar, de agir e de sentir. "Os valores organizacionais podem fortemente influenciar o que as pessoas realmente fazem", afirmam Deal e Kennedy (1988, p. 22). Não somente o comportamento do indivíduo é influenciado pelos valores, mas também o julgamento que ele faz do comportamento dos outros, pertinente ao sistema organizacional. Os valores têm a função "de vincular as pessoas, de modo que elas permaneçam dentro do sistema e executem as funções que lhes foram atribuídas" (Katz \& Kahn, 1978, p. 70). Os valores podem ser considerados como um projeto para a organização e um esforço para atingir as metas por ela fixadas. As normas organizacionais estão enraizadas nos valores e podem ser consideradas como uma operacionalização dos valores organizacionais. Os valores são mais abrangentes que as normas e constituem uma espécie de ideologia. As normas definem explicitamente as formas de comportamento esperado dos membros de uma organização e os valores "proporcionam uma justificação mais elabo- 
rada e generalizada, tanto para o comportamento apropriado, como para as atividades e funções do sistema" (Katz \& Kahn, 1978, p. 70).

4) Hierarquia dos valores. Um sistema de valores, segundo Rokeach (1968/69) é "nada mais do que uma disposição hierárquica de valores, uma classificação ordenada de valores ao longo de um continuum de importância" (p. 551). As pessoas, as organizações e as culturas se diferenciam entre si, não tanto pelo fato de possuir valores diferentes, mas pela organização hierárquica dos mesmos. Os valores organizacionais implicam necessariamente uma preferência, uma distinção entre o importante e o secundário, entre o que tem valor e o que não tem. Assim, a essência mesma dos valores organizacionais parece ser a de permitir a sua hierarquização (Tamayo, 2000). A organização hierárquica dos valores pressupõe que os membros da organização não se relacionam com o mundo físico e social como observadores que assistem a um espetáculo, mas como atores que participam, que tomam partido, que se envolvem nele, que transformam o ambiente organizacional e tentam se impor e se fazer reconhecer no meio social. Segundo Tamayo $(1994,1996)$ a idéia de graus de valor, de uma escala de valores ao longo de um contínuo de importância, encontra a sua base na relação dos valores com o tempo, elemento fundamental para o seu desenvolvimento, com a missão e os objetivos da empresa, cuja especificidade e importância organizacional impõem uma ordem de primazia, e com o esforço realizado pela empresa e pelos seus membros para a obtenção das metas propostas.

A fonte dos valores organizacionais é constituída por exigências da organização e dos indivíduos que a compõem. Essas exigências abrangem um leque que vai desde as necessidades biológicas dos indivíduos até as necessidades referentes à sobrevivência e ao bemestar da própria organização. A organização e os seus membros, para poder dar conta da realidade, têm que reconhecer essas necessidades e criar ou aprender respostas apropriadas para a sua satisfação. Através deste mecanismo, tanto a organização como os seus membros passam a representar de forma consciente essas necessidades como valores ou metas a serem atingidas. 
A estrutura axiológica de uma empresa pode ser descrita como um sistema relativamente estável de valores. Ela define a natureza das crenças e dos princípios que dominam na organização e o tipo de motivação característico da mesma. A percepção das prioridades axiológicas da organização por parte do empregado, é uma operação de tipo cognitivo que lhes permite desenvolver uma representação mental da organização, um modelo interno da empresa. O comportamento dos empregados na organização, as explicações que eles dão da rotina organizacional e, possivelmente, o comprometimento dos empregados com a empresa são determinados pela percepção que eles têm dos valores organizacionais.

A análise dos valores percebidos pelos empregados como sendo característicos da empresa fornece indícios sobre a forma como os objetivos gerais da mesma são captados pelos seus membros. A importância deste ponto não pode ser negligenciada. Modelos mentais diferentes provocam, inevitavelmente, percepções diferentes da empresa, do comportamento organizacional e das tarefas a serem executadas. Por exemplo, um simples feedback dado por um supervisor a um empregado, pode ser interpretado por este último, de forma não prevista nem desejada pelo primeiro, em função de diferenças, entre os dois, na percepção dos valores da empresa. Cada um deles pode ter uma visão diferente das prioridades axiológicas da organização. As formas de pensar, de refletir, de solucionar problemas, de comunicar com os colegas e com os gerentes, de analisar as expectativas próprias e as dos outros são fenômenos influenciados pelos valores compartilhados numa organização. Os valores, junto com os outros componentes da cultura organizacional, são determinantes do comportamento organizacional (Triandis, 1994).

Apesar da importância que têm os valores para a cultura e a vida organizacional, poucas tentativas tem sido realizadas para a sua avaliação sistemática (Bond, 1997). O esforço mais significativo foi realizado por Hofstede (1980). Em relação à avaliação dos valores organizacionais a partir da percepção dos empregados, os autores desta pesquisa encontraram um único instrumento psicométrico publicado. Ele foi elaborado no Brasil por Tamayo e Gondim (1996). Tra- 
ta-se de uma escala fatorial composta por 38 valores organizacionais distribuídos em cinco fatores. A obtenção dos valores para a escala foi realizada através de um levantamento com 113 empregados de cinco organizações públicas e privadas. Foram levantadas mais de 500 sugestões de valores, muitas delas idênticas e outras apresentando bastantes semelhanças. Através de análise de conteúdo, os autores chegaram a uma lista de 48 valores que eles consideraram como sendo uma amostra representativa dos valores organizacionais. Estes valores foram distribuídos num folheto de forma aleatória e administrados a uma amostra de 537 sujeitos de 16 empresas públicas e privadas. Os resultados foram submetidos a análise fatorial. Cinco fatores foram encontrados: eficácia/eficiência, interação no trabalho, gestão, inovação e respeito ao empregado. Os coeficientes alpha variaram de 0,70 a 0,91 .

Se já existe no país um instrumento de medida dos valores organizacionais, devidamente validado, porque construir mais um instrumento? Embora os parâmetros de validade e precisão da escala de Tamayo e Gondim (1996) não apresentem problemas que justifiquem a elaboração de um novo instrumento, considerou-se que a estratégia exclusivamente empírica utilizada para a construção da mesma, já que ela foi construída unicamente a partir de um levantamento de valores organizacionais realizado em várias empresas, podia ser complementada com uma abordagem teórica. Os estudos de Schwartz e Ros (1995) e Schwartz (1999) mostraram que os valores pessoais podem ser analisados nos níveis individual e cultural. Os autores postularam que as dimensões culturais dos valores expressam os problemas ou dilemas básicos encontrados pelas sociedades para regular a atividade humana. A análise no nível cultural, por eles proposta, considera três dimensões fundamentais que constituem os eixos da cultura da sociedade e expressam as soluções dadas aos problemas relativos à natureza da relação entre o indivíduo e do grupo, à forma de garantir o comportamento responsável necessário para sustentar a estrutura da sociedade e à relação da humanidade com o meio físico e social. A idéia para esta pesquisa foi utilizar estes postulados para estudar os valores organizacionais, considerando a organização como 
uma coletividade que encontra e deve solucionar os mesmos problemas fundamentais que qualquer sociedade.

Da mesma forma que o indivíduo e que as sociedades em geral, as organizações encontram exigências universais que têm que ser satisfeitas para garantir a sua sobrevivência. Com base na teoria e nos resultados acima citados, na construção deste instrumento postularase que toda organização enfrenta três problemas fundamentais:

1) A relação entre o indivíduo e o grupo; relação que é e será sempre conflituosa já que é difícil conciliar as metas e os interesses do indivíduo e do grupo que a constitui.

2) A necessidade de elaborar uma estrutura para garantir o funcionamento da organização. Para poder subsistir a organização tem que definir papéis, normas, subsistemas organizacionais, relações entre eles, estratégias de trabalho etc.

3) A relação da organização com o meio ambiente natural e social já que toda organização existe num ponto determinado do planeta e inserida numa sociedade concreta. Para poder subsistir, tem que interagir continuamente com o ambiente físico (por exemplo, extração de matéria-prima), com a sociedade e com outras organizações (Schwartz \& Ros, 1995).

Ao tentar dar resposta a estas exigências básicas a organização cria padrões de comportamento e valores que expressam os princípios que orientam a sua vida quotidiana. "Assim como a sociedade tem uma herança cultural”, afirmam Katz e Kahn (1978), "as organizações sociais possuem padrões distintos de sentimentos e crenças coletivos que são transmitidos aos novos membros do grupo" (p. 85).

A organização e os seus membros, para poder dar conta da realidade, têm que reconhecer essas necessidades básicas e aprender respostas apropriadas para a sua satisfação. Desta forma, tanto a organização como os seus membros passam a representar conscientemente essas necessidades como valores e metas a serem atingidas (Sagiv \& Schwartz, 1995). Segundo Rokeach (1973), os valores são representações cognitivas de necessidades e motivos, não somente de necessidades individuais, mas também de exigências sociais e culturais. Essas representações cognitivas são mediadoras entre as necessidades 
e as metas e as intenções. "Os valores desenvolvem um papel fundamental fornecendo um significado cognitivo às necessidades e transformando-as em metas e intenções comportamentais" (Erez, 1997, p. 205).

A partir das três necessidades acima mencionadas e com base na abordagem cultural dos valores (Schwartz \& Ros, 1995), postulam-se três dimensões bipolares para representar as alternativas de resposta das organizações: autonomia (individualismo) versus conservadorismo (coletivismo), hierarquia versus igualitarismo e domínio versus harmonia. Estas três dimensões constituem a base teórica imediata para a construção do Inventário de Valores Organizacionais.

\section{a) Autonomia versus Conservadorismo}

Um dos problemas fundamentais que têm que solucionar as organizações é o da relação entre o indivíduo e o grupo formado pela própria organização. As soluções apresentadas pelas organizações podem-se situar num contínuo definido pela autonomia e o conservadorismo. Algumas organizações podem enfatizar os interesses do grupo. Nelas, os interesses do indivíduo não são vistos como sendo diferentes dos do grupo. Os valores característicos aos interesses do grupo referem-se à necessidade de conservação dos usos, costumes, estruturas de poder, etc. Esses valores enfatizam a manutenção do status quo na organização, da interdição de comportamentos que possam perturbar as normas e as tradições da empresa. Os valores deste pólo são valores sociocêntricos. No extremo oposto desta dimensão, encontram-se valores importantes particularmente nas organizações que percebem o empregado como sendo uma entidade autônoma, habilitada para perseguir os seus próprios interesses e fixar as suas metas em harmonia com as metas e normas da organização. Essas organizações atribuem elevada importância à criatividade individual e valorizam a responsabilidade individual. Duas formas de autonomia podem ser identificadas: o individualismo ou autonomia intelectual que, centrado nos valores de autodeterminação (criatividade, curiosidade), garante ao indivíduo o direito de perseguir, na vida da organização, as suas próprias diretrizes intelectuais e 
o individualismo afetivo que enfatiza os valores de estimulação e hedonismo (prazer, vida excitante) e garante ao indivíduo o espaço para utilizar a sua experiência afetiva.

Em certas organizações, as pessoas são vistas como nascidas para desempenhar um papel determinado: o papel de chefe, o papel de subordinado e em outras, como indivíduos autônomos com direito para definir e conquistar, com base na sua competência pessoal, o seu próprio espaço na organização. Nas organizações que dão preferência aos valores relativos ao conservadorismo, a iniciativa e a criatividade do empregado não são promovidas porque a ênfase é dada às tradições e a conservação do status quo, aos caminhos e às soluções já conhecidas e testadas pela organização no passado. Nas organizações nas quais predominam os valores de autonomia, a ênfase é na inovação, na criatividade do indivíduo, no teste de novas soluções, de novas formas de pensar, de agir, de executar o trabalho e de produzir.

\section{b) Hierarquia versus Igualitarismo}

O segundo problema que enfrenta toda organização é o da estrutura. As organizações são necessariamente sistemas estruturados. Desta forma, o problema da estrutura é fundamental e sem a sua solução a organização não pode funcionar e nem sequer existir. A estrutura define o sistema social da organização, as funções que devem ser executadas e as relações entre as diversas unidades e entre os membros da empresa. As soluções dadas a este problema pelas diversas organizações situam-se num contínuo que vai da hierarquia ao igualitarismo. O primeiro pólo enfatiza a hierarquia como forma de garantir o comprometimento dos membros da empresa com a missão da mesma. Certos valores culturais (autoridade, poder social, influência, fiscalização, supervisão etc.) expressam uma preferência nítida pela hierarquia. Esses valores enfatizam a legitimidade da definição e distribuição hierárquica de recursos e de papéis sociais na organização. No outro pólo desta dimensão, encontra-se a opção pela estrutura igualitária que expressa a preocupação com o bem-estar dos outros, da organização em geral. Esta escolha implica poucos níveis de auto- 
ridade e a opção por um gerenciamento de tipo participativo. Cada um deles é reconhecido como qualificado e responsável no exercício de suas funções. Exemplos de valores culturais típicos da estrutura igualitária são a justiça social, a igualdade, a responsabilidade, a eqüidade.

c) Harmonia versus Domínio

Toda empresa existe num contexto geográfico e social e insere-se no mundo do mercado com características precisas e com concorrentes bem definidos. Em certos casos, ela pode precisar de matéria-prima para a elaboração dos seus produtos e de implantar sistemas para a eliminação de resíduos. Por outra parte, ela coexiste com outras empresas que, freqüentemente, visam a mesma clientela. Desta forma, ela é forçada a definir o seu tipo de relacionamento com o meio físico e social. Duas alternativas opostas, situadas num contínuo, definem as soluções da organização. Num dos pólos, pode ser representada a tentativa de afirmação assertiva da empresa através do domínio dos recursos materiais, do mercado, da tecnologia e do conhecimento na área específica de atuação. Os valores relativos a este fator referem-se ao domínio do ambiente físico e social através da auto-afirmação da empresa. Os valores deste pólo promovem a realização de esforços diretos para obter o controle e a exploração do meio ambiente, para satisfazer os interesses da organização e para dominar o mercado. No pólo oposto desta dimensão, encontram-se valores (por exemplo, proteção da natureza, cooperação, integração interorganizacional) que enfatizam a harmonia com a natureza e com as outras organizações. Eles expressam a tentativa de um acomodamento harmonioso no seu meio ambiente.

As organizações nas quais predominam os valores de domínio apresentam uma marcada tendência ao sucesso mesmo às custas de dimensões ecológicas do meio ambiente. A sua interação com a natureza pode ser violenta e desequilibrada. Além disso, as suas relações com outras organizações podem ser definidas principalmente pelo desejo de sucesso e de domínio e pela vontade nítida de impor os seus produtos e a sua imagem. As organizações que dão prioridade aos valores de harmonia procuram o desenvolvimento e o sucesso 
através de uma interação harmoniosa com a natureza e com as outras organizações, promovendo valores ecológicos, a paz, a tolerância e a cooperação (Barnett \& Carroll, 1987; Staber, 1992).

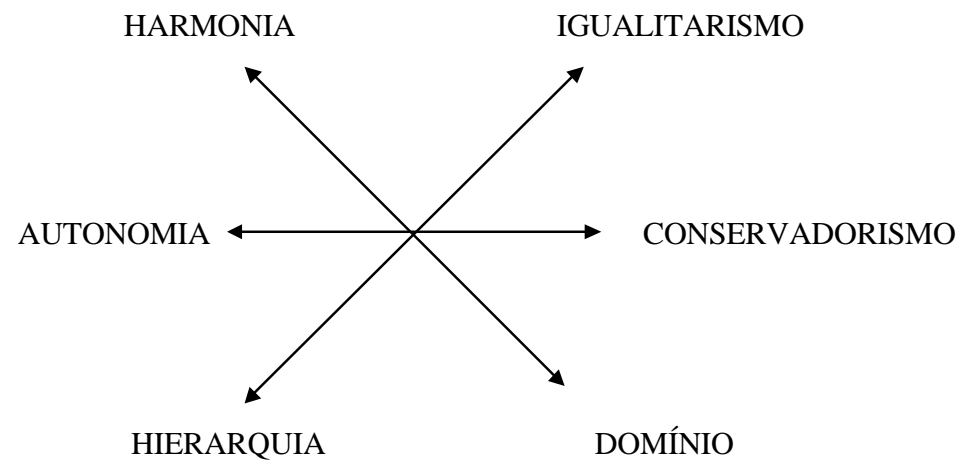

Figura 1. Estrutura teórica dos valores organizacionais

Pode-se postular que a multiplicidade de valores existentes nas diversas organizações se estrutura em torno de poucas motivações. Os valores organizacionais se organizam e se estruturam em torno das três dimensões bipolares, anteriormente mencionadas: autonomia versus conservadorismo, hierarquia versus igualitarismo, domínio versus harmonia. Desta forma, os valores organizacionais podem ser classificados em seis tipos motivacionais definidos pelos pólos destas três dimensões (Figura 1). Este foi o modelo utilizado para a construção deste novo instrumento.

\section{Método}

\section{Construção dos itens}

O Inventário de Valores Organizacionais integra duas abordagens diferentes: a empírica e a teórica. Os itens que haviam sido levantados empiricamente por Tamayo e Gondim (1996) para a construção da Escala de Valores Organizacionais foram utilizados para este novo ins- 
trumento, já que eles expressam adequadamente alguns dos construtos axiológicos que se pretendia medir, de acordo com o modelo teórico representado na Figura 1. Para representar de forma apropriada os tipos motivacionais de valores que não estavam suficientemente representados na escala de Tamayo e Gondim, foram construídos novos itens. Estes, foram construídos a partir dos pressupostos teóricos, procurando valores que expressassem adequadamente os pólos de igualitarismo e autonomia, que eram os menos representados na Escala de Tamayo e Gondim. Todos os itens foram colocados num folheto com instruções apropriadas e submetidos a análise semântica, realizada com três grupos de 6 a 8 empregados cada um, para verificar a clareza das instruções e a perfeita compreensão dos itens. Os resultados da análise semântica sugeriram modificações em quatro itens (por exemplo, mudança de uma palavra por outra mais familiar para os sujeitos). O inventário para validação ficou composto por 37 itens, seguidos de uma escala de 7 pontos (de 0 a 6 ).

\section{Amostra}

Para a sua validação o instrumento foi administrado a uma amostra de 1010 empregados voluntários de cinco organizações do Distrito Federal, com idade média de 38,82 anos ( $\mathrm{DP}=6,58)$, dos dois sexos ( $54 \%$ homens e $44 \%$ mulheres), sendo que $12 \%$ ocupavam o cargo de gerente e os outros empregados.

\section{Procedimento}

A administração do Inventário para sua validação foi realizada individualmente, nas próprias organizações, em locais apropriados para este tipo de atividade. A administração do Inventário foi realizada por três auxiliares de pesquisa, todos do sexo feminino.

\section{Análise dos dados}

Para a análise dos dados foi utilizado o SPSS (Statistical Package of Social Science). Os dados foram submetidos a uma análise multidimensional ALSCAL (Algorithmic Scaling), com duas dimensões e, posteriormente, com três dimensões, utilizando o modelo euclidiano. 
304 A.Tamayo et al.

\section{Resultados}

A análise multidimensional revelou as seguintes medidas de fit para a solução bidimensional: s-stress $=0,26224$, Stress $=0,23975$ e RSQ $($ Squared Correlation $)=0,80674$. A medida s-stress foi sugerida por Takane, Young, e Leeuw (1977) e está baseada no quadrado das distâncias entre os valores. No índice Stress de Kruskal (1964) são utilizadas as distâncias em vez do quadrado das mesmas. Estas duas medidas variam entre 1 (a pior solução) e 0 (fit perfeito). A RSQ consiste simplesmente no quadrado da correlação entre os dados e as distâncias. As medidas de fit obtidas nesta análise não são perfeitas, mas elas garantem que a solução encontrada é satisfatória.

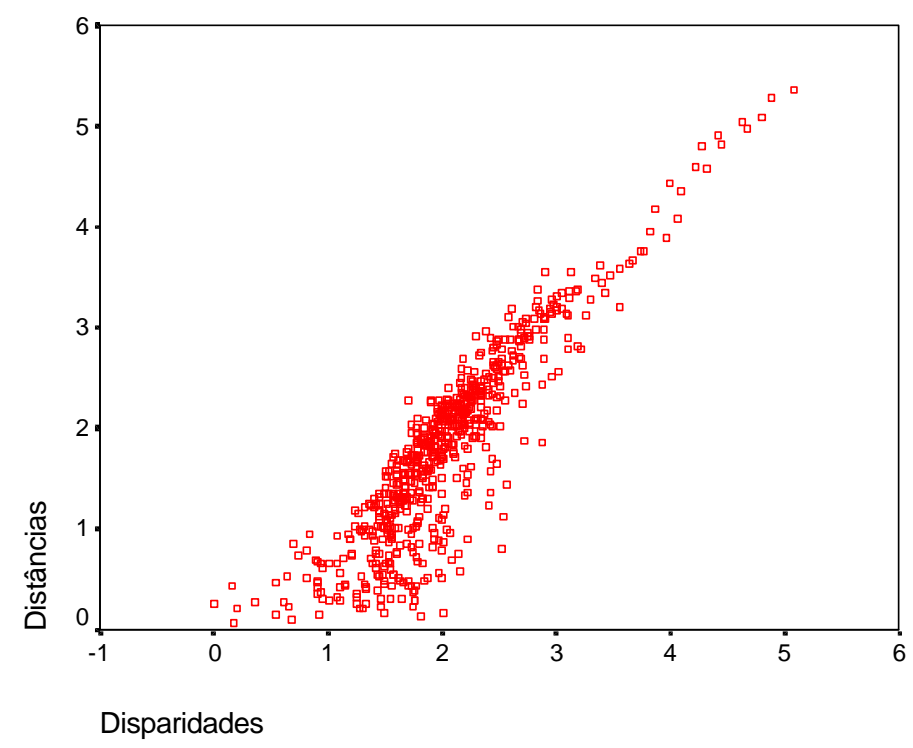

Figura 2. Modelo euclidiano das distâncias para a solução bi-dimensional

A Figura 2 apresenta o scatterplot of linear fit. Ele representa os dados brutos (eixo horizontal) versus as distâncias estabelecidas en- 
tre os valores a partir das respostas dos sujeitos (eixo vertical). Os dados brutos foram padronizados. As distâncias são as distancias euclidianas entre todos os valores. A transformação representada nesta figura é bastante linear e regular. Estas duas características permitem concluir que a solução encontrada é satisfatória, isto é, que as distâncias estão bem adaptadas aos dados.

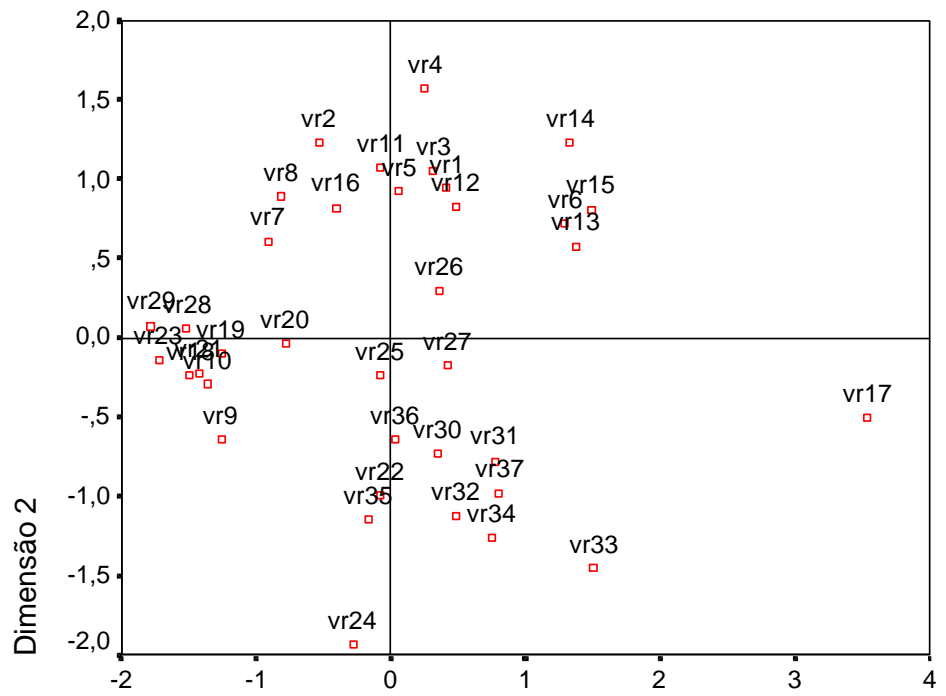

Dimensão 1

Figura 3. Solução bi-dimensional

A Figura 3 mostra a configuração dos itens no espaço semântico bidimensional. O item 17 foi o único que ocupou uma posição periférica, bastante distante dos outros, o qual significa que ele apresenta baixa comunalidade com os outros. Os valores assim distribuídos no espaço semântico formam conjuntos, relativamente homogêneos, que representam os pólos axiológicos postulados nesta pesquisa.

Foi também realizada uma análise multidimensional com três dimensões, com quatro iterações, sendo todos os outros parâmetros os 
mesmos da solução anterior. Os três indicadores da adequação (measures of fit) foram as seguintes: s-stress $=0,18319$, Stress $=0,15985$ e RSQ $=0,88563$. As três medidas de fit obtidas com a solução de três dimensões são melhores que as obtidas com duas dimensões, indicando que a primeira é mais adequada.

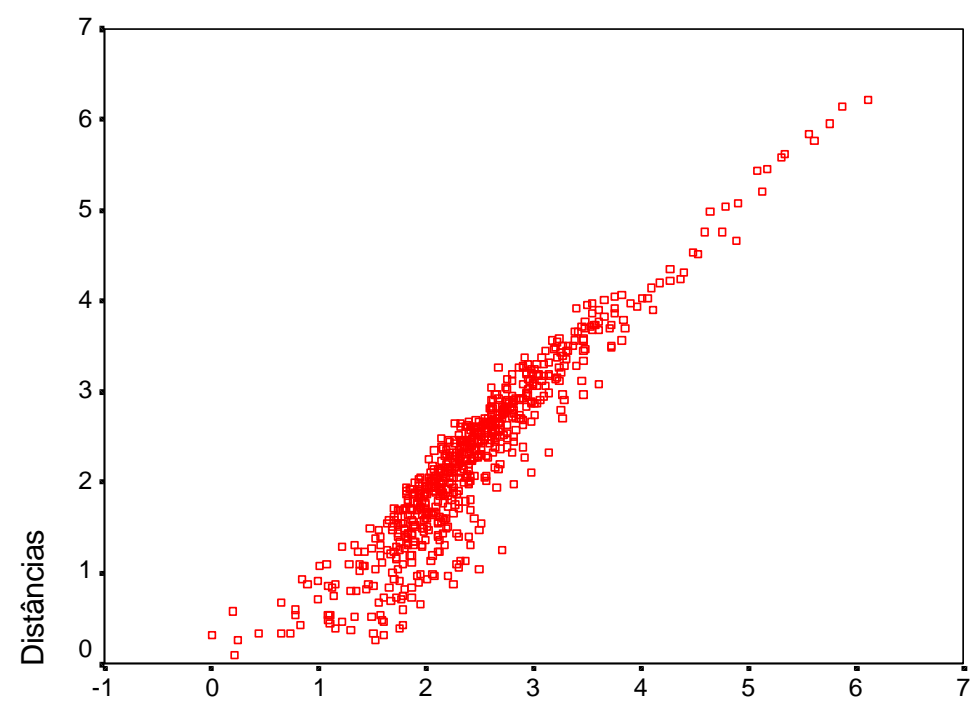

\section{Disparidades}

Figura 4. Modelo euclidiano das distâncias para a solução tridimensional

O scatterplot of linear fit, sintetizado pelas três medidas de fit encontra-se na Figura 4. A linearidade e a regularidade deste gráfico revelam que o instrumento utilizado é adaptado para a avaliação da percepção dos valores organizacionais nas empresas. Obviamente, esta adaptação não é perfeita, mas suficiente para garantir a validade do instrumento. 


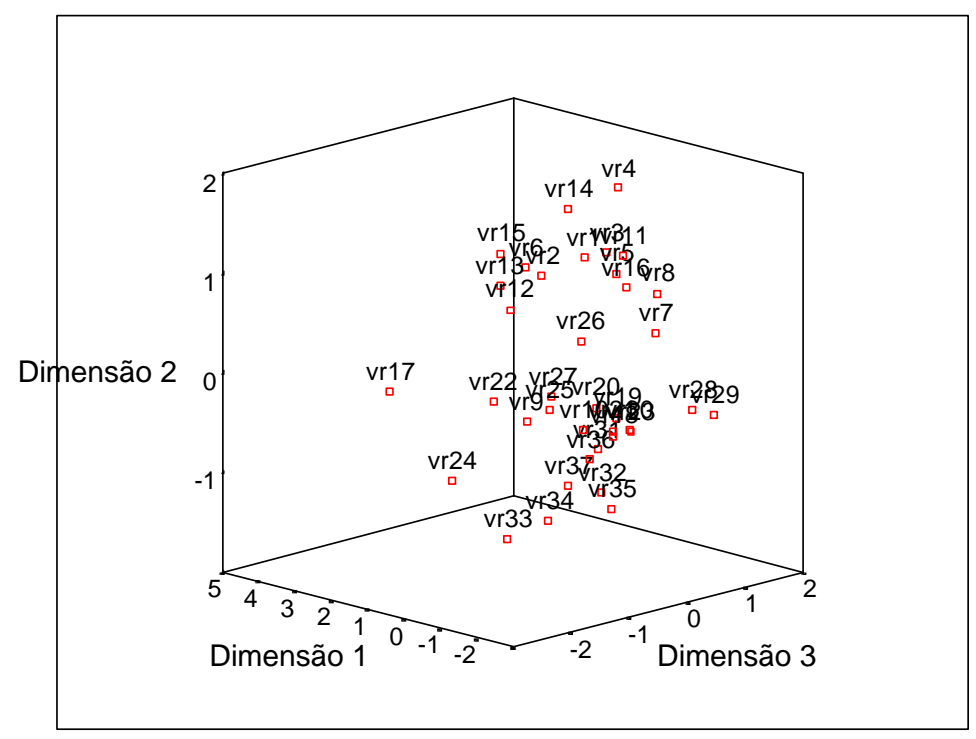

Figura 5. Modelo tri-dimensional

A Figura 5 apresenta a posição dos valores no espaço semântico multidimensional, para a solução tridimensional. Os coeficientes alpha foram calculados para cada um dos pólos das três dimensões. A Tabela 1 apresenta os itens que compõem cada um dos pólos bem como os coeficientes alpha. Os coeficientes alpha foram todos superiores a 0,83 com exceção do alpha para o tipo motivacional conservadorismo que foi de 0,77. O item 17 ("Participação dos empregados nos lucros da empresa") surgiu na análise multidimensional como componente do igualitarismo. Os resultados da análise de precisão indicaram que, com a sua eliminação, podia-se melhorar o coeficiente alpha. Como foi indicado anteriormente, este item destacou-se do conjunto dos itens, ocupando uma posição distante dos demais (Figura 3). Com base nestes resultados o item 17 foi eliminado. Desta forma, o Inventário de Valores Organizacionais ficou constituído por 36 valores numa escala de 7 pontos. 
Tabela 1

Itens correspondentes a cada um dos tipos motivacionais e coeficientes alpha

\begin{tabular}{llc}
\hline Tipos motivacionais & \multicolumn{1}{c}{ Itens } & Alpha \\
\hline Autonomia & $01,03,04,05$ & 0,83 \\
Conservadorismo & $02,07,08,11,16$ & 0,77 \\
Hierarquia & $09,10,17,18,19,20,22,24,27,28$ & 0,87 \\
Igualitarismo & $06,12,13,14,15,25,26$ & 0,85 \\
Domínio & $21,23,31,32,33,34,35,36$ & 0,84 \\
Harmonia & 29,30 & 0,85 \\
\hline
\end{tabular}

\section{Discussão}

As medidas de adaptação obtidas, tanto na solução bidimensional como na tridimensional, são satisfatórias indicando que a distribuição dos valores no espaço multidimensional e as distâncias entre eles estão bem adaptadas aos dados. Isto significa que é possível representar os valores organizacionais nas três dimensões postuladas, já que a distribuição dos valores no espaço multidimensional forma conjuntos que têm sentido psicológico e que expressam os pólos ou fatores axiológicos postulados: hierarquia, igualitarismo, autonomia, conservadorismo, domínio e harmonia (Tabela 1). Nem todos os itens, porém, se situaram no pólo esperado, observando-se a migração de alguns deles para um pólo diferente do esperado. Isto explica a desigualdade no número de itens compondo cada um dos pólos axiológicos. Este resultado é explicado pela interpretação dada pelos sujeitos a esses itens, diferente da esperada pelos pesquisadores.

Os pressupostos teóricos básicos foram confirmados, já que as três dimensões propostas foram encontradas. Os valores organizacionais ordenam-se em torno às três dimensões fundamentais postuladas. O antagonismo entre os pólos das dimensões foi confirmado parcialmente. Somente na dimensão "Hierarquia versus Igualitarismo" os pólos apareceram como sendo opostos. Os valores que os constituem situaram-se em regiões opostas, o qual significa que as suas metas são conflituosas. Os pólos das dimensões "Autonomia versus Conservadorismo" e "Domínio versus Harmonia" foram respectivamente adjacentes, manifestando que os valores com eles relacionados não são conflituosos no âmbito organizacional. Este 
resultado, apesar de não ser esperado na pesquisa, não é surpreendente e, possivelmente, constitui uma especificidade da cultura brasileira. Schwartz (1999) ao estudar com uma amostra de mais de 35.000 sujeitos a estrutura axiológica de 49 países constatou que o Brasil ocupa uma posição central no gráfico representando as semelhanças e as diferenças entre os 49 países nos pólos das três dimensões axiológicas. Esta posição central sugere que a oposição existente entre os pólos das três dimensões é praticamente anulada, permitindo a convivência ou coexistência pacífica na cultura brasileira de elementos antagônicos. Esta situação parece se refletir nas organizações utilizadas para a validação do IVO, já que em duas das dimensões não foi observada a oposição postulada entre os pólos. Desta forma, os valores que compõem os pólos de "Autonomia versus Conservadorismo" e de "Domínio versus Harmonia" não são conflituosos nas organizações. Nelas podem coexistir, sem conflito, metas de autonomia (inovação, criatividade, por exemplo) com metas de conservadorismo (tradição, supervisão) e metas de domínio com metas de harmonia.

Os coeficientes de precisão são satisfatórios. O alpha do fator "Conservadorismo" foi o mais baixo e mesmo assim ele é aceitável. Alguns autores consideram que a partir de 0,70 a precisão de um instrumento de medida pode ser considerada satisfatória. Outros, porém, exigem coeficientes a partir de 0,80 . O coeficiente de "Conservadorismo" está perto deste limite.

Os parâmetros psicométricos obtidos permitem concluir que a validação do Inventário de Valores Organizacionais foi bem estabelecida. Em conseqüência, ele pode ser utilizado em pesquisa, em diagnósticos organizacionais, em intervenções e em estudos da cultura da empresa. Do ponto de vista da correção do IVO convém salientar que o escore em cada um dos fatores se obtém pelo somatório dos valores escalares atribuídos pelos sujeitos a cada um dos itens do fator dividido pelo número de itens no fator considerado (Tabela 1).

Os seis tipos motivacionais do IVO avaliam valores compartilhados que expressam crenças fundamentais sobre o que é bom, correto e desejável numa organização. Eles permitem identificar componentes 
da cultura organizacional e diferenciar organizações ou unidades organizacionais a partir de seis aspectos fundamentais da vida organizacional: (1) a importância atribuída à procura de inovação, de criatividade, e de independência na execução do trabalho (Autonomia); (2) a ênfase na interdependência dos membros da organização e na obtenção de objetivos grupais (Conservadorismo); (3) a relevância dos papéis hierárquicos na estruturação do relacionamento interpessoal e na distribuição de recursos e de poder (Hierarquia); (4) a procura efetiva do bem-estar de todos os membros da organização e da sua participação nos processos decisórios (Igualitarismo); (5) a importância dada ao sucesso organizacional e à satisfação dos clientes (Domínio) e (6) o respeito à natureza e a procura de complementariedade organizacional (Harmonia).

O Inventário de Valores Organizacionais oferece também a possibilidade de realizar a avaliação em cada um dos tipos motivacionais em dois níveis diferentes: dos valores reais e dos valores desejados (ver Anexo). A expressão valores reais refere-se aos valores que, na percepção dos empregados, são realmente praticados na empresa e à força que eles têm na vida quotidiana da organização. A expressão valores desejados designa a prioridade ou grau de importância que os empregados gostariam que fosse dada a cada um dos valores apresentados no IVO. Desta forma, o IVO permite avaliar as prioridades axiológicas reais e desejadas da organização. A grande vantagem desta dupla avaliação é que ela permite estabelecer índices de satisfação/insatisfação dos empregados com as prioridades axiológicas da empresa em cada um dos seis tipos motivacionais acima descritos.

\section{Referências}

Allport, F. H. (1962). The structuring of events: outline of a general theory with applications to psychology. Psychological Review, 61, 281-303.

Barnett, W., \& Carroll, G. (1987). Competition and mutualism among early telephone companies. Administrative Science Quarterly, 32, 400-421.

Bond, M. H. (1997). Adding values to the cross-cultural study of organizational behavior. Reculer pour mieux sauter. In P. C. Earley \& M. Erez (Orgs.), New perspectives on International Industrial/Organizational Psychology (pp. 256275). San Francisco: The New Lexington Press. 
Davis, S. M. (1984). Managing corporate culture. Cambridge: Ballinger.

Deal, T., \& Kennedy, A. (1988). Corporate cultures. The rites and rituals of corporate life. London: Penguin.

De Freitas, M. E. (1994). Cultura organizacional. Formação, tipologias e impactos. São Paulo: Makron.

Erez, M. (1997). A culture-based model of work motivation. In P. C. Earley \& M. Erez (Orgs.), New perspectives on International Industrial/Organizational Psychology (pp. 194-242). San Francisco: The New Lexington Press.

Hofstede, G. (1980). Culture's consequences: international differences in workrelated values. Beverly Hills: Sage.

Hofstede, G., Neuijen, B., Ohayv, B. B., \& Sanders, G. (1990). Measuring organizational cutures: A qualitative and quantitative study across twenty cases. Administrative Science Quarterly, 35, 286-316.

Kabanoff, B., \& Daly, J. P. (2000). Values espoused by Australian and US organisations. Applied Psychology: An International Review, 49 (2), 284314.

Kabanoff, B., Waldersee, R., \& Cohen, M. (1995). Espoused values and organizational change themes. Academy of Management Journal, 38, 10751104.

Katz, D., \& Kahn, R. L. (1978). Psicologia social das organizações. São Paulo: Atlas.

Kotter, J. P., \& Heskett, J. L. (1994). A cultura corporativa e o desempenho empresarial. São Paulo: Makron.

Kluckhon, C. (1951). Values and value orientations in the theory of action: An exploration in definition and classification. In T. Parsons \& E. Shils (Orgs.), Toward a general theory of action (pp.388-433). Cambridge: Harvard University Press.

Kruskal, J. B. (1964). Nonmetric multidimensional scaling. Psychometrika, 29, $1-27$.

Peters, T., \& Waterman, Jr. R. (1982). In search of excellence. New York: Harper \& Row.

Rokeach, M. (1973). The nature of human values. New York: Free Press.

Rokeach, M. (1968/69). The role of values in public opinion research. Public Opinion Quarterly, 32, 547-559.

Rowlinson, M., \& Hassard, J. (1993). The invention of corporate culture: A history of the histories of Cadbury. Human Relations, 46, 299-326.

Sagiv, L., \& Schwartz, S. H. (1995). Value priorities and readiness for out-group social contact. Journal of Personality \& Social Psychology, 69 (3), 437448 .

Schwartz, S. H. (1994). Are there universal aspects in the structure and contents of human values? Journal of Social Issues, 50, 19-45. 
Schwartz, S. H. (1999). A theory of cultural values and some implications for work. Applied Psychology: An International Review, 48 (1), 23-47.

Schwartz, S. H., \& Ros, M. (1995). Values in the west: A theoretical and empirical challenge to the individualism-collectivism cultural dimension. World Psychology, 1, 91-122.

Schwartz, S. H., \& Bilsky, W. (1987). Toward a universal psychological structure of human values. Journal of Personality and Social Psychology, 53, 550-562.

Schwartz, S. H., \& Bilsky, W. (1990). Toward a theory of the universal content and structure of values: extensions and cross-cultural replications. Journal of Personality and Social Psychology, 58, 878-891.

Schein, E. H. (1965). Organizational Psychology. Englewood Cliffs: Printice-Hall.

Schein, E. H. (1990). Organizational culture. American Psychologist, 45, 109 119.

Sego, D. J., Hui, C., \& Law, K. S. (1997). Operationalizing cultural values as the mean of individual values. Problems and suggestions for research. In P. C. Earley \& $\quad$ M. Erez (Orgs.), New perspectives on international industrial/organizational psychology. (pp. 148-159). San Francisco: The New Lexington Press.

Staber, U. H. (1992). Organizational interdependence and organizational mortality in the cooperative sector: A community ecology perspective. Human Relations, 45, 1191-1212.

Takane, Y., Young, F. W., \& de Leeuw, J. (1977). Nonmetric individual differences multidimensional scaling: An alternating least squares method with optimal scaling features. Psychometrika, 42, 7-67.

Tamayo, A. (2000). Prioridades axiológicas e satisfação no trabalho. Revista de Administração, 35 (2), 37-47.

Tamayo, A. (1998). Valores organizacionais: sua relação com satisfação no trabalho, cidadania organizacional e comprometimento afetivo. Revista de Administração, 33 (3), 56-63.

Tamayo, A. (1996). Valores organizacionais. In A. Tamayo, J. E. Borges-Andrade, \& W. Codo (Orgs.), Trabalho, Organizações e Cultura (pp. 175-193). São Paulo: Cooperativa de Autores Associados.

Tamayo, A. (1994). Hierarquia de valores transculturais e brasileiros. Psicologia: Teoria e Pesquisa, 10, 269-285.

Tamayo, A., \& Gondim, M. G. C. (1996). Escala de valores organizacionais. Revista de Administração, 31 (2), 62-72.

Tamayo, A., \& Schwartz, S. H. (1993). Estrutura motivacional dos valores humanos. Psicologia: Teoria e Pesquisa, 9, 329-348.

Triandis, H. C. (1994). Culture and social behavior. New York: McGraw-Hill.

Triandis, H. C. (1995). Individualism \& Collectivism. San Francisco: Westview Press. 
Alvaro Tamayo, doutor em Psicologia Social pela Université de Louvain, Bélgica, é professor titular da Universidade de Brasília; Ana Magnólia Mendes, é doutora em Psicologia Organizacional pela Universidade de Brasília, é professora adjunta da Universidade de Brasília; Maria das Graças T. da Paz, doutora em Psicologia Organizacional pela Universidade de Brasília, é diretoria do Instituto de Psicologia da Universidade de Brasília. Endereço para correspondência: Alvaro Tamayo, SHIN, QI 3, Conjunto 4, Casa 2, 71.505-240, Brasília, DF.

E-mail: tamayo@unb.br.
Sobre os autores 


\section{ANEXO \\ INVENTARIODE VALORES ORGANIZACIONAIS}

Este questionário traz uma lista de itens que expressam valores da organização. Sua tarefa é avaliar quão importantes são esses valores como princípios orientadores da vida da sua organização. Esta avaliação deve ser feita a dois níveis:

Real: quanto cada valor é praticado na realidade atual da sua organização.

Desejável: quanto cada valor deveria ser importante para sua organização.

Para dar sua opinião, utilize uma escala de 0 a 6 , conforme abaixo:

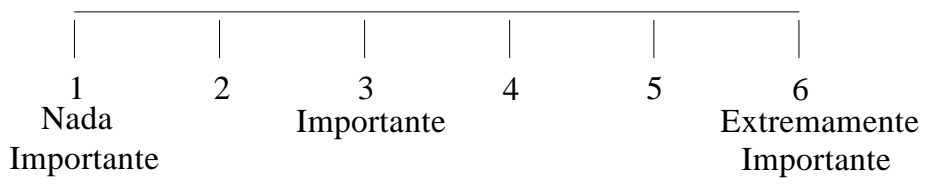

Lembre-se de que quanto mais próximo do 6 mais importante é o valor.

Coloque um círculo em torno do número escolhido para cada um dos aspectos - Real e Desejáve 1- na coluna correspondente.

Não há resposta certa ou errada. Responda de acordo com o seu entendimento e interpretação. Não deixe nenhum item em branco.

Agradecemos a sua colaboração. Não é necessário se identificar. 


\section{Item}

1. Capacidade de inovar na organização

2. Capacidade de realizar as tarefas sem necessidade de supervisão constante

3. Introdução de novidades no trabalho

4. Abertura para expor sugestões e opiniões sobre o trabalho

5. Busca constante de informação e novidades

6. Continuidade de políticas e projetos organizacionais

7. Fidelidade à organização

8. Segurança de pessoas e bens

9. Preservação dos costumes vigentes da organização

10. Tradição de respeito às ordens

11. Clima de ajuda mútua

12. Distribuição do poder pelos diversos níveis

13. Tratamento proporcional ao mérito

14. Oportunidades iguais para todos os empregados

15. Imparcialidade nas decisões administrativas

16. Clima de relacionamento amistoso entre os empregados

17. Respeito às pessoas com cargo de chefia

18. Respeito das regras e normas

estabelecidas pela organização

19. Controle do serviço executado

20. Respeito aos níveis de autoridade

21. Capacidade de influenciar pessoas na organização

22. Preocupação com o cumprimento de horários e compromissos

23.

Dificuldade de alterar regras, normas e comportamentos na organização

24. Acompanhamento e avaliação contínuos das tarefas

25. Ambiente de relacionamento

interorganizacional adequado

26. Complementariedade de papéis entre organizações

27. Utilização de recursos sem causar danos ao meio ambiente

28. Proteção ao meio ambiente

29. Intercâmbio com outras organizações

30. Atuação conjunta com outras empresas

\section{Real}

\begin{tabular}{|l|l|l|l|l|l|l|}
\hline 0 & 1 & 2 & 3 & 4 & 5 & 6 \\
\hline
\end{tabular}

\begin{tabular}{|l|l|l|l|l|l|l|}
\hline 0 & 1 & 2 & 3 & 4 & 5 & 6 \\
\hline
\end{tabular}

\begin{tabular}{l|l|l|l|l|l|l|}
\hline 0 & 1 & 2 & 3 & 4 & 5 & 6 \\
\hline
\end{tabular}

$\begin{array}{lllllll}0 & 1 & 2 & 3 & 4 & 5 & 6\end{array}$

$\begin{array}{llllllll}0 & 1 & 2 & 3 & 4 & 5 & 6\end{array}$

$\begin{array}{lllllllll}0 & 1 & 2 & 3 & 4 & 5 & 6\end{array}$

\begin{tabular}{llllllll|}
\hline 0 & 1 & 2 & 3 & 4 & 5 & 6 \\
\hline
\end{tabular}

\begin{tabular}{l|l|l|l|l|l|l|}
\hline 0 & 1 & 2 & 3 & 4 & 5 & 6 \\
\hline
\end{tabular}

$\begin{array}{llllllll}0 & 1 & 2 & 3 & 4 & 5 & 6\end{array}$

\begin{tabular}{llllllll|}
\hline 0 & 1 & 2 & 3 & 4 & 5 & 6 \\
\hline
\end{tabular}

\begin{tabular}{lllllllll|}
\hline 0 & 1 & 2 & 3 & 4 & 5 & 6 \\
\hline
\end{tabular}

$\begin{array}{llllllll}0 & 1 & 2 & 3 & 4 & 5 & 6\end{array}$

\begin{tabular}{l|l|l|l|l|l|l|}
\hline 0 & 1 & 2 & 3 & 4 & 5 & 6 \\
\hline
\end{tabular}

$\begin{array}{llllllll}0 & 1 & 2 & 3 & 4 & 5 & 6\end{array}$

\begin{tabular}{|lllllllll|}
\hline 0 & 1 & 2 & 3 & 4 & 5 & 6 \\
\hline
\end{tabular}

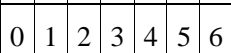

\begin{tabular}{llllllll|}
\hline 0 & 1 & 2 & 3 & 4 & 5 & 6 \\
\hline
\end{tabular}

$\begin{array}{llllllll}0 & 1 & 2 & 3 & 4 & 5 & 6\end{array}$

\begin{tabular}{llllllll|}
\hline 0 & 1 & 2 & 3 & 4 & 5 & 6 \\
\hline
\end{tabular}

\begin{tabular}{llllllll|}
0 & 1 & 2 & 3 & 4 & 5 & 6 \\
\hline
\end{tabular}

$\begin{array}{llllllll}0 & 1 & 2 & 3 & 4 & 5 & 6\end{array}$

$\begin{array}{llllllll}0 & 1 & 2 & 3 & 4 & 5 & 6\end{array}$

$\begin{array}{llllllll}0 & 1 & 2 & 3 & 4 & 5 & 6\end{array}$

\begin{tabular}{|llllll|l|l|}
\hline 0 & 1 & 2 & 3 & 4 & 5 & 6
\end{tabular}

$\begin{array}{llllllll}0 & 1 & 2 & 3 & 4 & 5 & 6\end{array}$

\begin{tabular}{|lll|l|l|l|l|}
\hline 0 & 1 & 2 & 3 & 4 & 5 & 6
\end{tabular}

$\begin{array}{lllllllll}0 & 1 & 2 & 3 & 4 & 5 & 6\end{array}$

\begin{tabular}{llllllll}
0 & 1 & 2 & 3 & 4 & 5 & 6 \\
\hline & 1 & 2 & 3 & 4 & 5 & 6
\end{tabular}

\begin{tabular}{|lll|l|l|l|l|}
\hline 0 & 1 & 2 & 3 & 4 & 5 & 6 \\
\hline
\end{tabular}

\begin{tabular}{|l|l|l|l|l|l|l|}
\hline 0 & 1 & 2 & 3 & 4 & 5 & 6 \\
\hline
\end{tabular}
Desejável

\begin{tabular}{|l|l|l|l|l|l|l|}
\hline 0 & 1 & 2 & 3 & 4 & 5 & 6 \\
\hline & & & & & & \\
\end{tabular}

$\begin{array}{llllllll}0 & 1 & 2 & 3 & 4 & 5 & 6\end{array}$

\begin{tabular}{lllllllll}
\hline 0 & 1 & 2 & 3 & 4 & 5 & 6 \\
\hline
\end{tabular}

$\begin{array}{llllllll}0 & 1 & 2 & 3 & 4 & 5 & 6\end{array}$

$\begin{array}{llllllll}0 & 1 & 2 & 3 & 4 & 5 & 6\end{array}$

$\begin{array}{llllllll}0 & 1 & 2 & 3 & 4 & 5 & 6\end{array}$

$\begin{array}{lllllllll}0 & 1 & 2 & 3 & 4 & 5 & 6\end{array}$

\begin{tabular}{llllllll}
\hline 0 & 1 & 2 & 3 & 4 & 5 & 6 \\
\hline
\end{tabular}

$\begin{array}{llllllll}0 & 1 & 2 & 3 & 4 & 5 & 6\end{array}$

\begin{tabular}{llllllll|}
\hline 0 & 1 & 2 & 3 & 4 & 5 & 6 \\
\hline
\end{tabular}

$\begin{array}{llllllll}0 & 1 & 2 & 3 & 4 & 5 & 6\end{array}$

$\begin{array}{llllllll}0 & 1 & 2 & 3 & 4 & 5 & 6\end{array}$

\begin{tabular}{l|l|l|l|l|l|l|}
\hline 0 & 1 & 2 & 3 & 4 & 5 & 6 \\
\hline
\end{tabular}

$\begin{array}{lllllll}0 & 1 & 2 & 3 & 4 & 5 & 6\end{array}$

$\begin{array}{llllllll}0 & 1 & 2 & 3 & 4 & 5 & 6\end{array}$

$\begin{array}{llllllll}0 & 1 & 2 & 3 & 4 & 5 & 6\end{array}$

\begin{tabular}{llllllll}
\hline 0 & 1 & 2 & 3 & 4 & 5 & 6 \\
\hline
\end{tabular}

$\begin{array}{llllllll}0 & 1 & 2 & 3 & 4 & 5 & 6\end{array}$

\begin{tabular}{llllllll}
\hline 0 & 1 & 2 & 3 & 4 & 5 & 6 \\
\hline & 1 & 2 & 3 & 4 & 5 & 6 \\
\hline
\end{tabular}

\begin{tabular}{|lll|l|l|l|l|}
\hline 0 & 1 & 2 & 3 & 4 & 5 & 6 \\
\hline
\end{tabular}

$\begin{array}{lllllll}0 & 1 & 2 & 3 & 4 & 5 & 6\end{array}$

$\begin{array}{llllllll}0 & 1 & 2 & 3 & 4 & 5 & 6\end{array}$

$\begin{array}{lllllll}0 & 1 & 2 & 3 & 4 & 5 & 6\end{array}$

$\begin{array}{lllllll}0 & 1 & 2 & 3 & 4 & 5 & 6\end{array}$

$\begin{array}{llllllll}0 & 1 & 2 & 3 & 4 & 5 & 6\end{array}$

$\begin{array}{lllllll}0 & 1 & 2 & 3 & 4 & 5 & 6\end{array}$

$\begin{array}{lllllll}0 & 1 & 2 & 3 & 4 & 5 & 6\end{array}$

$\begin{array}{llllllll}0 & 1 & 2 & 3 & 4 & 5 & 6\end{array}$

\begin{tabular}{llllllll|}
\hline 0 & 1 & 2 & 3 & 4 & 5 & 6 \\
\hline
\end{tabular}

\begin{tabular}{lllllll}
\hline 0 & 1 & 2 & 3 & 4 & 5 & 6 \\
\hline
\end{tabular} 